Natura@economía

ISSN 2226-9479 (Versión electrónica)

Website: http://revistas.lamolina.edu.pe/index.php/neu

\title{
Distribución de mamíferos según rango altitudinal en la Reserva Nacional de Huascarán
}

\section{Distribution of mammals according to the range of altitude in the Nation Reserve of Huascaran}

\author{
Martin Palomino-C. ${ }^{1 *}$; Yiem Ataucusi ${ }^{1}$
}

\begin{abstract}
${ }^{1}$ Doctorado en Economía de los Recursos Naturales y el Desarrollo Sustentable, Facultad de Economía y Planificación, Universidad Nacional Agraria La Molina. Av. La Molina s/n -Ciudad Universitaria, Lima, Perú. Email: mpalomino@lamolina.edu.pe
\end{abstract}

Recibido: 28 abril del 2019; Aceptado: 20 junio del 2019

\section{Resumen}

El Estado peruano reconoce espacios continentales y/o marinos como áreas naturales protegidas por su importancia para la conservación de la biodiversidad. Para ello, se asigna un presupuesto anual inferior al beneficio obtenido por la exportación de productos relacionados con la diversidad biológica y los recursos naturales. Una manera de explicar la riqueza de las especies está en función de los factores geográficos según las ciencias biológicas. Por ello, el presente estudio analiza los más relevantes estudios sobre la distribución de los mamíferos (mediano y grande) según rangos altitudinales, para luego determinar estadísticamente la abundancia de las especies en el Parque Nacional de Huascarán. Se utiliza el meta-análisis y el instrumento de simulación de Monte Carlo, se demuestra que en altitudes intermedias se encuentran la mayor diversidad de mamíferos.

Palabras clave: meta-análisis: rango altitudinal; Monte Carlo; promedio altitudinal.

\begin{abstract}
The Peruvian State recognizes continental and or marine spaces as natural protected areas due to their importance for the conservation of biodiversity. To this end, an annual budget is allocated lower than the benefit obtained from the export of products related to biological diversity and natural resources. One way to explain the richness of the species is based on geographical factors, according to the biological sciences. Therefore, the present study analyzes the most relevant studies on the distribution of mammals (medium and large) according to altitudinal ranges, and then statistically determine the abundance of the Huascaran National Park. The meta-analysis and the Monte Carlo simulation instrument are used, it is shown that the intermediate diversity of mammals is found at intermediate altitudes.
\end{abstract}

Keywords: meta-analysis: altitudinal range; Monte Carlo; altitudinal average.

Forma de citar el artículo: Palomino-C \& Ataucusi. 2019. Distribución de mamíferos según rango altitudinal en la Reserva Nacional de Huascarán. Natura@economía 4(1): 38-52 (2019). 


\section{Introducción}

Las áreas naturales en el Perú están sometidas a diversas presiones antrópicas (Póveda, 2006) tales como: cacería ilegal, sobrepastoreo, acumulación de desechos sólidos, actividades turísticas descontroladas, introducción de especies exóticas, incendios, fragmentación del suelo, pérdida y degradación del hábitat, sobreexplotación, contaminación, cambios en los ciclos geoquímicos y el cambio climático (Polasky et al., 2005), los cuales están vulnerando el hábitat de la flora y fauna. En los mamíferos el cambio es más evidente por ser una especie altamente sensible a las perturbaciones antropogénicas y a las variaciones ambientales (GarcíaBurgos et al., 2014; Rumiz et al., 1998). A razón de esto, el Estado peruano protege las áreas naturales, asignando un presupuesto anual; sin embargo, son insuficientes en relación a los beneficios que obtiene la economía nacional por la exportación de productos relacionados con la diversidad biológica y recursos naturales (León, 2007).

La ciencia biológica explica la riqueza de las especies a partir de los factores geográficos según altitud, latitud y profundidad, determinando que la composición de las especies, cambia según elevación, ello hace que la diversidad disminuya más de lo normal conforme aumenta la altitud, sin embargo, la mayor diversidad se encuentra en altitudes intermedios (Martín-Piera, 1999; Rowe, 2009; Ferro \& Barquez, 2014).

Los mamíferos son importantes en el ecosistema debido a que son: i) indicadores de calidad ambiental, su presencia indica el buen estado de conservación de un hábitat, ii) controladores biológicos, regulan la presencia de presas como roedores, insectos y aves, y iii) reguladores del ecosistema, ayudan a mantener el ecosistema mediante la dispersión de semillas (Crooks \& Soulé, 1999; Cossíos et al., 2007; Prugh et al., 2009).

\section{$\underline{\text { Rango altitudinal }}$}

Se refiere a la altitud sobre el nivel del mar hasta la cima, lugar con heterogeneidad de características ambientales, que se refleja en la composición y riqueza de flora y fauna (Ferro \& Barquez, 2014). Según el geógrafo peruano Pulgar (1981), el Perú tiene ocho regiones naturales: Chala, Yunga, Quechua, Suni, Puna, Janca o Cordillera, Rupa Rupa o Selva alta, Omagua o Selva baja, cada región natural tiene aspectos en común como el clima, suelo, altitud, relieve, flora y la fauna. Mientras Brack-Egg (1986), toma en consideración diferentes factores ecológicos: tipos de clima, regiones geográficas, hidrografía, flora y fauna, identificando la existencia de once ecorregiones en el Perú. Ambos consideran a la fauna como uno de los criterios de distribución. La diferencia es que Pulgar (1981) considera una mayor variación de pisos altitudinales en la sierra.

\section{Mamíferos medianos y grandes}

Los mamíferos, son vertebrados que poseen glándulas mamarias a través de las cuales la hembra alimenta a sus crías con su propia leche (RAE), proceso que no sucede en los reptiles o las aves. Además, los mamíferos poseen pelo o piel a diferencia de los reptiles, peces y aves que tienen escamas o plumas respectivamente. Por otro lado, los mamíferos viven a partir del consumo de oxígeno y de la producción de dióxido de carbono que es exhalado al ambiente en el cual habitan. Los mamíferos son clasificados entre tres grandes tipos de animales: aquellos que ponen huevos como el ornitorrinco, los marsupiales (que se caracterizan por contar con una especie de bolsa donde llevar a las crías, tales como el canguro o el koala) y los que nacen de placenta (los animales que se desarrollan hasta nacer en medio de la placenta). Los mamíferos son los animales de mayor atracción, por su característica física, comportamiento $\mathrm{y}$ son fáciles de 
percibir, mientras que los reptiles y las aves son los animales menos favoritos, porque generan fobia, son aburridos y difíciles de observar (Carr, 2016).

Se consideran como mamíferos medianos y grandes aquellos con más de $500 \mathrm{~g}$ de peso (Tlapaya \& Gallina, 2010; MonroyVilchis et al., 2011; García-Burgos, 2007), por su alta sensibilidad a las perturbaciones antropogénicas que responden a variaciones ambientales, resaltando que en el país son poco estudiados a nivel de distribución en gradientes altitudinales, la mayoría de las investigaciones están enfocados a pequeños mamíferos y voladores (García-Burgos et al., 2014; Rumiz et al., 1998).

Mamíferos según rango altitudinal. Martín-Piera (1999), Rowe (2009) y Ferro \& Barquez (2014) mencionan que la distribución de mamíferos cambia según la elevación, eso hace que la diversidad disminuya conforme aumenta la altitud. Sin embargo, la mayor diversidad se encuentra en altitudes intermedias.

El objetivo de la presente investigación fue reunir los más importantes estudios realizados sobre la distribución de mamíferos en rangos altitudinales, según tamaño (mediano y grande), en el Parque Nacional de Huascarán.

\section{Materiales y métodos}

El estudio se realizó en base a la información del Parque Nacional Huascarán (Figura 1), localizado en la zona norte-centro del territorio peruano, en el departamento de Ancash, con una superficie de 340 mil hectáreas (ha), por presentar un rango altitudinal entre 2400 y 6768 m.s.n.m.; además, según consideraciones descritas por Pulgar (1981) cuenta con las regiones Quechua, Suni, Puna y Janca (SERNANP, 2011).

En base a estudios previos, existen esfuerzos significativos de la comunidad científica por estudiar la distribución de mamíferos en diferentes investigaciones publicados en la última década. En la Figura 2 se muestra que desde el año 2005 se ha incrementado considerablemente en el número de investigaciones sobre distribución de mamíferos medianos y grandes mayores.

El análisis se realizó mediante el instrumento del meta-análisis, descritos por Werenkraut (2010), que consiste en la recopilación de información documental (Zorrilla, 1993), utilizado por el gran potencial para la sistematización de producción científica disponible y determinar patrones de información (Nijkamp et al., 2008); para la investigación se recurrió a bases de datos:

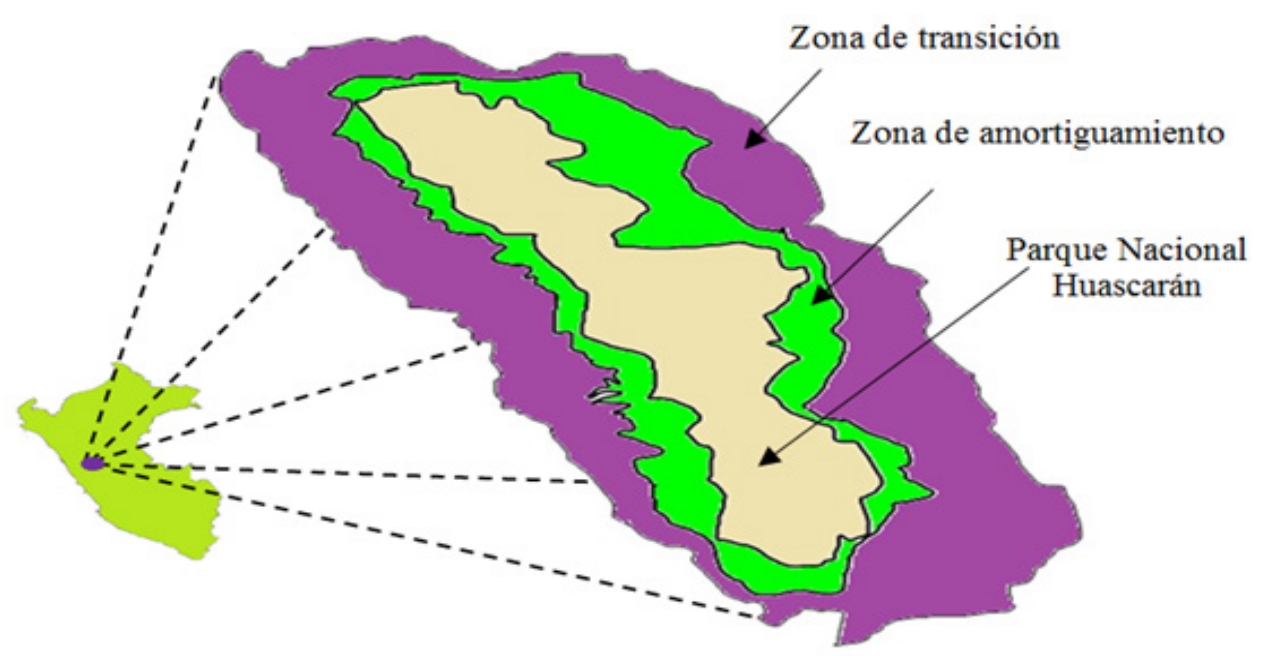

Figura 1: Localización del Parque Nacional Huascarán 
Scopus, Scielo y Latindex, información oficial y análisis de datos históricos del Parque Nacional de Huascarán, tales como los planes maestros, informes del Servicio Nacional de Áreas Naturales Protegidas por el Estado (SERNANP), reporte de The IUCN Red List of Threatened Species. Mientras que, para evaluar la probabilidad de abundancia según rangos altitudinales se realizó mediante la simulación de Monte Carlo con el uso del software Risk Simular versión Prueba.

El análisis estadístico, se utilizó para determinar la probabilidad de la altitud media de abundancia de especies, para luego agrupar en rangos altitudinales (Figura 3), según consideraciones descritas por Pulgar (1981).

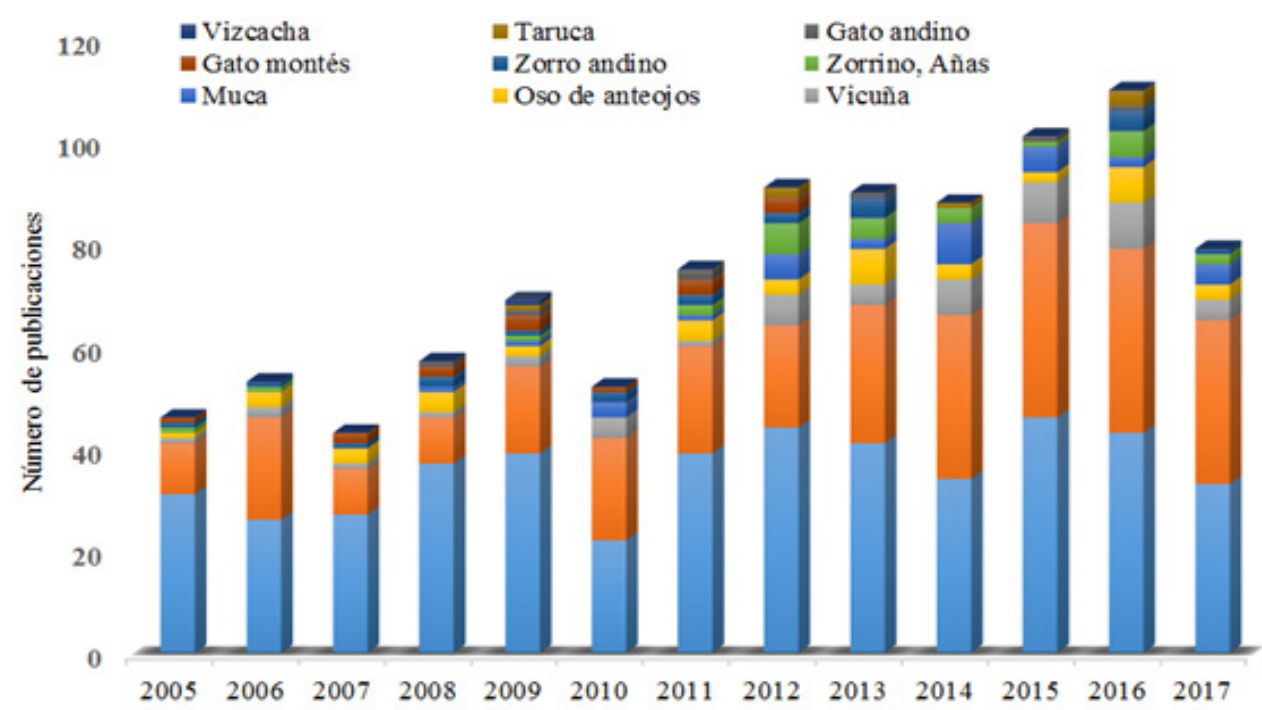

Figura 2: Publicaciones de artículos científicos acerca de la distribución de mamíferos medianos y mayores a $500 \mathrm{~g}$

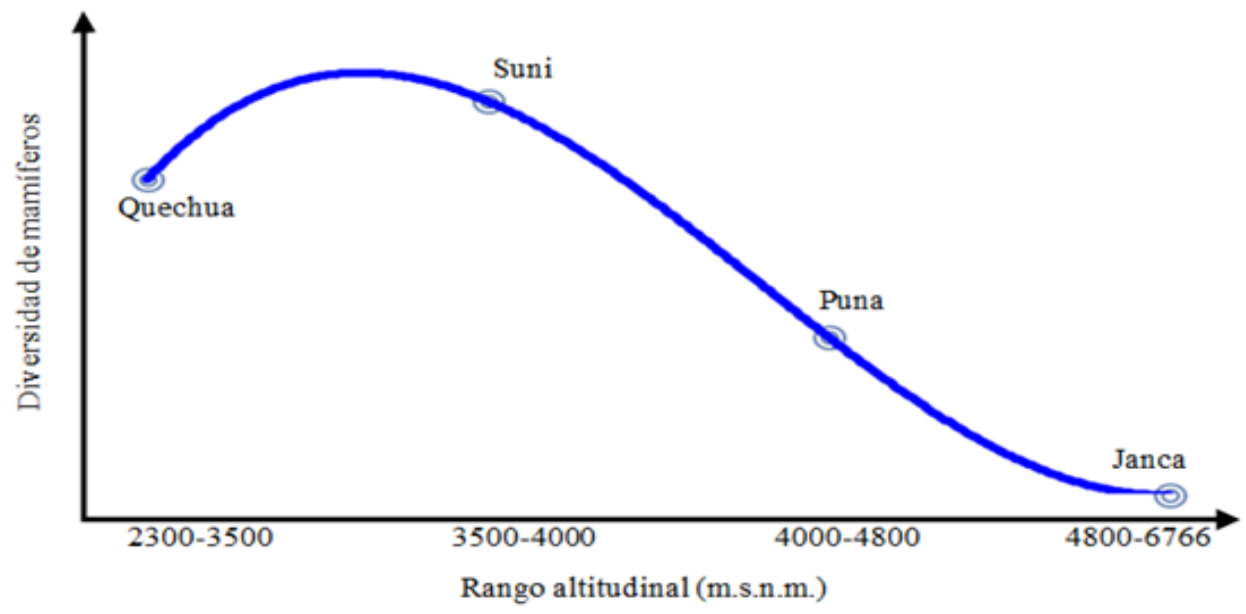

Figura 3: Distribución en rango altitudinal de mamíferos 


\section{Resultados y discusión}

En el Parque Nacional de Huascarán, se identificaron 18 mamíferos (SERNANP, 2011), de los cuales siete son mamíferos pequeños (roedores, murciélagos y marsupiales) y 11 están conformados por mamíferos medianos y grandes superiores a 500 g. La investigación se limita a analizar a los mamíferos medianos y grandes (Tabla 1). m.s.n.m.), con presencia muy reducida en la Yunga y Suni. Mientras que, la Taruca $(58,03 \%)$, Gato andino $(65,95 \%)$, Zorrino $(46,82 \%)$, Zorro andino $(60,30 \%)$ y Puma $(72,10 \%)$ habitan en el rango altitudinal Suni (3500-4000 m.s.n.m.), con presencia reducida en la Quechua y Puna. Por otra parte, la Vizcacha $(57,09 \%)$ y la Vicuña $(52,40 \%)$ habitan en el rango altitudinal

Tabla 1. Estado de conservación de mamíferos medianos y grandes

\begin{tabular}{|c|c|c|c|}
\hline \multirow[b]{2}{*}{ Nombre común } & \multirow[b]{2}{*}{ Nombre científico } & \multicolumn{2}{|c|}{ Categoría de Conservación } \\
\hline & & $\begin{array}{c}\text { D.S. No004-2014- } \\
\text { MINAGRI }\end{array}$ & IUCN \\
\hline Gato montés & Oncifelis colocolo & $\mathrm{DD}$ & NT \\
\hline Muca & Didelphis marsupialis & & $\mathrm{LC}$ \\
\hline Oso de anteojos & Tremarctos ornatus & & VU \\
\hline Taruca & Hippocamelus antisensis & & VU \\
\hline Vizcacha & Lagidium peruanum & & $\mathrm{LC}$ \\
\hline Gato andino & Leopardus jacobita & EN & $\mathrm{EN}$ \\
\hline Vicuña & Vicugna vicugna & NT & $\mathrm{LC}$ \\
\hline Zorrino, Añas & Conepatus chinga & & $\mathrm{LC}$ \\
\hline Venado cola blanca & Odocoileus virginianus & & $\mathrm{LC}$ \\
\hline Zorro andino & Pseudalopex culpaeus & & $\mathrm{LC}$ \\
\hline Puma & Puma concolor & NT & $\mathrm{LC}$ \\
\hline
\end{tabular}

De la meta-análisis realizada a partir de especies individuales (Anexo 1) se evaluó la probabilidad de abundancia, según rangos altitudinales, mediante la simulación de Monte Carlo con el uso del software Risk Simular versión Prueba. En la Tabla 2, se muestra el promedio de 10 mil interacciones aleatorias que se realizó en el ordenador, y el resultado estadístico de la Tabla 2 se sintetiza del Anexo 2.

Para de agrupación de mamíferos en rango altitudinal, se consideró la distribución planteada por Pulgar (1981). Los resultados demuestran de que el Gato montés $(97,71 \%)$, Muca $(95,26 \%)$, Oso de anteojos $(98,81 \%)$ y venado de cola blanca $(88,21 \%)$ habitan en el rango altitudinal Quechua (2300-3500
Puna (4000-4800 m.s.n.m.), con presencia significativa en Suni y con presencia reducida en Quechua y Janca.

En la Figura 4, se determinó los patrones de abundancia de las especies según rango altitudinal para el Parque Nacional de Huascarán; en el rango altitudinal Quechua (2300-3500 m.s.n.m.) se observa cuatro especies de mamíferos; mientras en rango altitudinal Suni (3500-4000 m.s.n.m.) se observa cinco especies de mamíferos; finalmente, en el rango altitudinal Puna (4000-4 800 m.s.n.m.) solo se observan dos especies de mamíferos, con ello se demuestra que la diversidad de especies disminuye más de lo normal conforme aumenta la altitud, sin embargo, la mayor diversidad se encuentra en altitud intermedia. 
Tabla 2: Probabilidad de distribución de mamíferos según piso altitudinal

\begin{tabular}{|c|c|c|c|c|c|c|c|c|}
\hline \multirow{2}{*}{$\begin{array}{l}\text { Nombre } \\
\text { común }\end{array}$} & \multirow{2}{*}{$\begin{array}{l}\text { Nombre } \\
\text { científico }\end{array}$} & \multirow{2}{*}{$\begin{array}{c}\text { Altitud } \\
\text { Media } \\
\text { m.s.n.m. }\end{array}$} & \multicolumn{6}{|c|}{$\begin{array}{l}\text { Rango altitudinal (m.s.n.m.) donde abunda cada especie de } \\
\text { mamíferos }\end{array}$} \\
\hline & & & $\begin{array}{c}\text { Yunga } \\
(500-2300)\end{array}$ & $\begin{array}{l}\text { Quechua } \\
(2300-3500)\end{array}$ & $\begin{array}{c}\text { Suni } \\
(3500-4000)\end{array}$ & $\begin{array}{c}\text { Puna } \\
(4000-4800)\end{array}$ & $\begin{array}{c}\text { Janca } \\
(4800-6768)\end{array}$ & Acum. \\
\hline Gato montés & $\begin{array}{l}\text { Oncifelis } \\
\text { colocolo }\end{array}$ & 2911,43 & $1,03 \%$ & $97,71 \%$ & $1,27 \%$ & $0,00 \%$ & $0,00 \%$ & $100 \%$ \\
\hline Muca & $\begin{array}{l}\text { Didelphis } \\
\text { marsupialis }\end{array}$ & 2740,00 & $4,65 \%$ & $95,26 \%$ & $0,10 \%$ & $0,00 \%$ & $0,00 \%$ & $100 \%$ \\
\hline $\begin{array}{l}\text { Oso de } \\
\text { anteojos }\end{array}$ & $\begin{array}{l}\text { Tremarctos } \\
\text { ornatus }\end{array}$ & 2657,14 & $1,19 \%$ & $98,81 \%$ & $0,00 \%$ & $0,00 \%$ & $0,00 \%$ & $100 \%$ \\
\hline Taruca & $\begin{array}{l}\text { Hippocamelus } \\
\text { antisensis }\end{array}$ & 3742,86 & $0,00 \%$ & $21,44 \%$ & $58,03 \%$ & $20,52 \%$ & $0.03 \%$ & $100 \%$ \\
\hline Vizcacha & $\begin{array}{l}\text { Lagidium } \\
\text { peruanum }\end{array}$ & 4062,57 & $0,00 \%$ & $3,78 \%$ & $38,32 \%$ & $57,09 \%$ & $0,94 \%$ & $100 \%$ \\
\hline Gato andino & $\begin{array}{l}\text { Leopardus } \\
\text { jacobita }\end{array}$ & 3832,73 & $0,00 \%$ & $9,24 \%$ & $65,95 \%$ & $24,82 \%$ & $0,00 \%$ & $100 \%$ \\
\hline Vicuña & Vicugna vicugna & 4009,09 & $0,00 \%$ & $2,31 \%$ & $45,27 \%$ & $52,40 \%$ & $0,04 \%$ & $100 \%$ \\
\hline Zorrino & $\begin{array}{l}\text { Conepatus } \\
\text { chinga }\end{array}$ & 3550,00 & $0,00 \%$ & $44,78 \%$ & $46,82 \%$ & $8,41 \%$ & $0,00 \%$ & $100 \%$ \\
\hline $\begin{array}{l}\text { Venado cola } \\
\text { blanca }\end{array}$ & $\begin{array}{l}\text { Odocoileus } \\
\text { virginianus }\end{array}$ & 3259,58 & $0,00 \%$ & $88,21 \%$ & $11,79 \%$ & $0,00 \%$ & $0,00 \%$ & $100 \%$ \\
\hline Zorro andino & $\begin{array}{l}\text { Pseudalopex } \\
\text { culpaeus }\end{array}$ & 3637,50 & $0,00 \%$ & $30,91 \%$ & $60,30 \%$ & $8,80 \%$ & $0,00 \%$ & $100 \%$ \\
\hline Puma & Puma concolor & 3654,00 & $0,00 \%$ & $23,25 \%$ & $72,10 \%$ & $4,66 \%$ & $0,00 \%$ & $100 \%$ \\
\hline
\end{tabular}

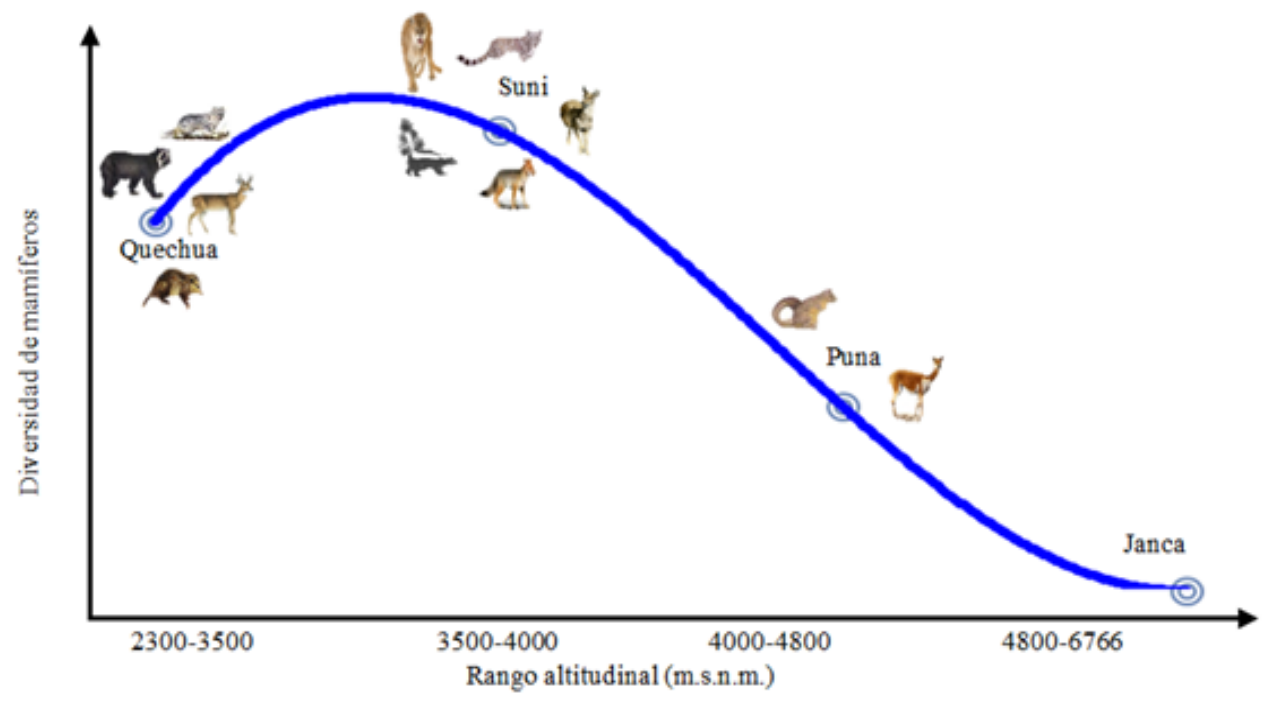

Figura 4: Distribución de mamíferos en rango altitudinal de mamíferos 


\section{Conclusiones}

Del estudio realizado se concluye que, en altitudes intermedias se encuentran la mayor diversidad de mamíferos. Siendo el Gato montés $(97,71 \%)$, Muca $(95,26 \%)$, Oso de anteojos $(98,81 \%)$ y Venado de cola blanca $(88,21 \%)$ los que habitan en el rango altitudinal Quechua (2300-3500 m.s.n.m.); mientras, la Taruca $(58,03 \%)$, Gato andino $(65,95 \%)$, Zorrino $(46,82 \%)$, Zorro andino $(60,30 \%)$ y Puma $(72,10 \%)$ habitan en el rango altitudinal Suni (3500-4000 m.s.n.m.) y que, la Vizcacha $(57,09 \%)$ y la Vicuña $(52,40 \%)$ habitan en el rango altitudinal Puna (4000-4800 m.s.n.m.). Por último, se recomienda aplicar la valoración económica de los mamíferos según rangos altitudinales a fin de justificar la conservación del área natural.

\section{Literatura citada}

Aguilar, J.M. 2011. Determinación de la variabilidad genética en tres poblaciones de vicuñas (Vicugna vicugna mensalis) en cautiverio a partir de muestras de heces. Tesis, Universidad Nacional Mayor de San Marcos, Facultad de Ciencias Biológicas, Lima. Disponible en http://200.62.146.130/handle/ cybertesis/1218

Albuja, L.H. 2007. Biología y Ecología del Venado de Cola Blanca (Odocoileus virginianus ustus Gray, 1874) en un sector de páramo. Biología, 7, 34-57. Disponible en http://bibdigital.epn.edu. ec/handle/15000/4746

All, J.; Medler, M.; Arques, S.; Cole, R.; Woodall, T.; King, J. \& Schmitt, C. 2017. Fire response to local climate variability: Huascarán National Park, Peru. Fire Ecology 13(2): 85-104. doi:10.4996/ fireecology.130288764

Arias-Alzate, A.; Delgado, C.A. \& Navarro, J.F. 2016. Nuevos registros de simpatría de Nasua nasua y Nasuella olivacea (Carnivora: Procyonidae) en el. Mammalogy Notes | Notas Mastozoológicas 3(1): 49-53.

Astua de Moraes, D.; Lew, D.; Costa, L. \& Pérez-Hernandez, R. 2016. Didelphis marsupialis. The IUCN Red List of Threatened Species 2016: e.T40501A22176071. doi:http://dx.doi. org/10.2305/IUCN.UK.2016-1.RLTS. T40501A22176071.en

Baldi, R.; Acebes, P.; Cuéllar, E.; Funes, M.; Hoces, D.; Puig, S. \& Franklin, W. 2016. Lama guanicoe. The IUCN Red List of Threatened Species 2016: e.T11186A18540211. doi:http://dx.doi. org/10.2305/IUCN.UK.2016-1.RLTS. T11186A18540211.en

Barberena, R.; Gil, A.; Neme, G.; Zangrando, F.; Politis, G.; Borrero, L. \& Prates, L. 2010. Ecología isotópica de guanaco (Lama guanicoe) en el sur de Sudamérica: tendencias espaciales, temporales e implicaciones arqueológicas. Zooarqueología a principios del siglo XXI. Aspectos teóricos, metodológicos y casos de estudio. 345-357. Disponible en https:// s3.amazonaws.com/academia.edu. documents/42535109/Ecologa_isotpica_ de_guanaco_Lama_guanic 20160210 26459-15tdy38.pdf?AWSAccessKeyId= AKIAIWOWYYGZ2Y53UL3A\&Expi res $=1502067622 \&$ Signature=PucE92e 7 GfUUie0ULawu56Y8orM\%3D\&respon se-content-disposition=inline $\%$

Barrio, J. 2006. Manejo no intencional de dos especies de cérvidos por exclusión de ganado en la parte alta del Parque Nacional Río Abiseo, Perú. Revista Electrónica Manejo de Fauna Silvestre en Latinoamérica 1(2): 1-10.

Barrio, J. \& Ferreyra, N. 2008. Hippocamelus antisensis. The IUCN Red List of Threatened Species 2008: e.T10053A3156943. doi:http://dx.doi. org/10.2305/IUCN.UK.2008.RLTS. T10053A3156943.en 
Bernal, N. 2016. Lagidium viscacia. The IUCN Red List of Threatened Species 2016: e.T11148A22190789. doi:http:// dx.doi.org/10.2305/IUCN.UK.2016-2 . RLTS.T11148A22190789.en

Brack-Egg, E. 1986. Las ecorregiones del Perú. boletín de Lima 44: 57-70.

Briones, H.Y.; Torgler, H.R. \& Álvarez, J.L. 2015. Desde el ojo de la cámara trampa. Consorcio Purús-Manu: WWF, CARE Perú, ProNaturaleza, ProPurús, Sociedad Zoológica de Fráncfort, ORAU. Lima, noviembre de 2015. Disponible en http://purusmanu.pe/wp-content/ uploads/2016/02/libro_fauna-FINAL. pdf

Cafrune, M.M.; Rebuffi, G. E.; Cabrera, R.H. \& Aguirre, D.H. 1996. Fasciola hepatica en llamas (Lama glama) de la Puna Argentina. Vet. Arg, 13(128): 570-574. Disponible en http://helminto. inta.gob.ar/Fasciola/Fasc $\% 20$ Hep $\% 20$ en $\% 20$ Llamas.PDF

Cajal, J.L. 1989. Uso de hábitat por vicuñas y guanacos en la Reserva de Biósfera San Guillermo. Vida Silvestre Neotropical 2: 21-31. Disponible en http://www.unesco. org.uy/geo/fileadmin/ciencias $\% 20$ naturales/mab/puna10.pdf

Carr, N. 2016. An analysis of zoo visitors' favourite and least favourite animals. Tourism Management Perspectives 20: 70-76. doi:10.1016/j.tmp.2016.07.006

Castillo, D.; Chávez, V.; Hoces, R.; Casas, A.; Rosadio, A. \& Wheeler, J. C. 2008. Contribución al estudio del parasitismo gastrointestinal en guanacos (Lama guanicoe cacsilensis). Revista de Investigaciones veterinarias del Perú 19(2): 168-175.

Chimento, N.R.; Derguy, M.R. \& Hemmer, H. 2014. Puma (Herpailurus) pumoides (Castellanos, 1958) nov. comb: Comentarios sistemáticos y registro fósil. Serie correlación geológica 30(2): 92-134.
Clavijo,A.\& Ramírez, G.F.2009. Taxonomía, distribución y estado de conservación de los felinos suramericanos: revisión monográfica. Boletín Científico Centro de Museos. Museo de Historia Natural 13: 43-60.

Colléony, A.; Clayton, S.; Couvet, D.; Saint Jalme, M. \& Prévot, A.C. 2017. Human preferences for species conservation: Animal charisma trumps endangered status. Biological Conservation 206: 263 269. doi:10.1016/j.biocon.2016.11.035

Cossíos, D.; Saavedra, F.B.; Bennet, M.; Bernal, N.; Fajardo, U., Lucherini, M. \& Perovic, P. 2007. Manual de metodologías para relevamientos de carnívoros alto andinos. Buenos Aires, Argentina.

Crooks, K.R. \& Soulé, M.E. 1999. Mesopredator release and avifaunal extinctions in a fragmented system. Nature 400(6744): 563.

Del Moral, J.F.; Zenteno, R. \& Lameda, F.I. 2009. Análisis biostático de las tensiones actuantes en el dentario del oso andino (Tremarctos ornatus), durante la mordedura. Acta zoológica mexicana 25(3): 551-567.

Díaz, I.A.; Sarmiento, C.; Ulloa, L.; Moreira, R.; Navia, R.; Véliz, E. \& Peña, C. 2002. Vertebrados terrestres de la Reserva Nacional Río Clarillo, Chile central: representatividad y conservación. Revista chilena de historia natural 75(2): 433-448. doi:http://dx.doi.org/10.4067/ S0716-078X2002000200013

Dourojeanni, M.J. 1968. Consideraciones sobre las Interinfluencias entre la Fauna, su Manejo y la Zootecnia, con Referencia Especial al Perú. Revista Forestal del Perú 2(1): 1-14.

Emmons, L.; Schiaffini, M. \& Schipper, J. 2016. Conepatus chinga. The IUCN Red List of Threatened Species 2016: e.T41630A45210528. doi:http://dx.doi. org/10.2305/IUCN.UK.2016-1.RLTS. 


\section{T41630A45210528.en}

Encina, R.L. \& Iglesias, A.P. 2009. Osteometría de Vicugna vicugna Molina, 1782 en el Pleistoceno final de Patagonia meridional chilena: Implicancias paleoecológicas y biogeográficas. Revista del Museo de Antropología 2(1): 127-140.

Fajardo, U.; Cossíos, D. \& Pacheco, V. 2014. Dieta de Leopardus colocolo (Carnivora: Felidae) en la Reserva Nacional de Junín, Junín, Perú. Revista peruana de biología 21(1): 061-070.

Ferro, I. \& Barquez, R.M. 2014. Patrones de distribución de micromamíferos en gradientes altitudinales del noroeste Argentino. Revista mexicana de biodiversidad 85(2): 472-490. doi:10.7550/rmb.38029

Figueroa, J. 2012. Presencia del oso andino Tremarctos ornatus (Carnivora: Ursidae) en el bosque tropical amazónico del Perú. Acta zoológica mexicana 28(3): 594-606.

Figueroa, J. 2013. Revisión de la dieta del oso andino Tremarctos ornatus (Carnivora: Ursidae) en América del Sur y nuevos registros para el Perú. Revista del Museo Argentino de Ciencias Naturale 15(1): $1-27$.

Figueroa, J. 2015. Interacciones humanooso andino Tremarctos ornatus en el Perú: consumo de cultivos y depredación de ganado. Therya 6(1): 251-278.

Figueroa, J. 2016. Ecología y conservación del oso andino (tremarctos ornatus) en las áreas naturales protegidas del perú. Universidad de Alicante. Obtenido de http://rua.ua.es/dspace/ handle/10045/59005\#vpreview

Gallina, S. \& Lopez Arevalo, H. 2016. Odocoileus virginianus. The IUCN Red List of Threatened Species 2016: e.T42394A22162580. doi:http://dx.doi.
org/10.2305/IUCN.UK.2016-2.RLTS. T42394A22162580.en

Gálvez, B.G. 1991. Revisión biogeográfica de los marsupiales del Parque Nacional del Manú, Perú . Espacio y Desarrollo, 3, 6-27. Disponible en http://revistas.pucp. edu.pe/index.php/espacioydesarrollo/ article/view/7887/8165

García-Burgos, J. 2007. Comparación de la riqueza de mamíferos medianos en un gradiente de manejo de cafetales del centro de Veracruz. Doctoral dissertation. Disponible en http:/cdigital.uv.mx/ handle/123456789/3696

García-Burgos, J.; Gallina, S. \& GonzálezRomero, A. 2014. Relación entre la riqueza de mamíferos medianos en cafetales y la heterogeneidad espacial en el centro de Veracruz. Acta zoológica mexicana 30(2): 337-356

Garzón-Jarrin, R.; García-Díaz, J.R.; \& Pérez-Bello, A. 2016. Valores de referencia para los parámetros hematológicos en el venado cola blanca (Odocoileus virginianus ustus) del Parque Nacional Cotopaxi. 38(2): 93-99.

Goldstein, I.; Velez-Liendo, X.; Paisley, S. \& Garshelis, D. 2008. Tremarctos ornatus. The IUCN Red List of Threatened Species 2008: e.T22066A9355162. doi:http://dx.doi.org/10.2305/IUCN. UK.2008.RLTS.T22066A9355162.en

Gómez-Puerta, L.A.; Ticona, D.S.; LópezUrbina, M.T. \& González, A.E. 2009. The Andean hog-nosed skunk Conepatus chinga Molina, 1782 as a new definitive host for Spirometra erinacei Faust, Campbell \& Kellog, 1929. Veterinary parasitology 3: 334-336. doi:https://doi. org/10.1016/j.vetpar.2008.11.030

Huamán, E. 2008. Composición de la dieta del guanaco (Lama guanicoe) en la comunidad de Huallhua y anexosAyacucho. Universidad Nacional Agraria La Molina. Disponible en http:// 
repositorio.lamolina.edu.pe/handle/ UNALM/1661

Iriarte, J.A.; Rau, J.R.; Villalobos, R.; Lagos, N. \& Sade, S. 2013. Revisión Actualizada Sobre La Biodiversidad y Conservación de los Felinos Silvestres de Chile. Boletín de Biodiversidad de Chile 8: 5-24.

Jayat, J.P.; Barquez, R.M.; Díaz, M.M.; \& Martinez, P.J. 1999. Aportes al conocimiento de la distribución de los carnívoros del noroeste de Argentina. Mastozoología Neotropical 6(1): 15-30.

Jiménez, J.E.; Yáñez, J.L.; Tabilo, E. L. \& Jaksic, F.M. 1996. Nichecomplementarity of South American foxes: reanalysis and test of a hypothesis. Revista Chilena de Historia Natural 69: 113-123. Obtenido de https://s3.amazona

Jimenez, P.C.; Villasante, J.F.; Talavera, B.C.; \& Villegas, L.N. 2006. Ecosistemas de Arequipa. Oferta Ambiental y Desarrollo Sostenible. Zonas Áridas 7(1): 118-132.

Lagos, N.; Villalobos, R. \& Iriarte, A. 2012. Nuevos registros de poblaciones de chinchilla de cola corta, Chinchilla chinchilla (Rodentia, Chinchillidae) en la cordillera de la Región de Atacama. Boletín del Museo de Historia Natural (Chile) 61: 191-196.

Lavariega, M.C.; Martin-Regalado, N. \& Gómez-Ugalde, R.M. 2012. Mamíferos del centro-occidente de Oaxaca, México. Therya 3(3): 350-370. doi:http://dx.doi. org/10.12933/therya-12-93.

León, F. 2007. El aporte de las áreas naturales protegidas a la economía nacional.

Lichtenstein, G.; Baldi, R.; Villalba, L.; Hoces, D.B. \& Laker, J. 2008. Vicugna vicugna. The IUCN Red List of Threatened Species 2008: e.T22956A9402796. doi:http://dx.doi. org/10.2305/IUCN.UK.2008.RLTS. T22956A9402796.en
Lucherini, M. 2016. Lycalopex culpaeus. The IUCN Red List of Threatened Species 2016: e.T6929A85324366. doi:http://dx.doi.org/10.2305/IUCN. UK.2016-1.RLTS.T6929A85324366.en

Lucherini, M.; Eizirik, E.; de Oliveira, T.; Pereira, J. \& Williams, R. 2016. Leopardus colocolo. The IUCN Red List of Threatened Species 2016: e.T15309A97204446. doi:http://dx.doi. org/10.2305/IUCN.UK.2016-1.RLTS. T15309A97204446.en

Martín-López, B.; Montes, C. \& Benayas, J. 2007. The non-economic motives behind the willingness to pay for biodiversity conservation. Biological conservation 139(1): 67-82. doi:10.1016/j. biocon.2007.06.005

Martín-Piera, F. 1999. Apuntes sobre biodiversidad y conservación de insectos: dilemas, ficciones y¿ soluciones? Boletín de la Sociedad entomológica Aragonesa 20: 25-55.

Medina, C.; Zeballos, H. \& López, E. 2012. Diversidad de mamíferos en los bosques montanos del valle de Kcosnipata, Cusco, Perú. Mastozoología neotropical 19(1): 85-104.

Monroy-Vilchis, O.; Zarco-González, M. M.; Ramírez-Pulido, J. \& AguileraReyes, U. 2011. Diversidad de mamíferos de la Reserva Natural Sierra Nanchititla, México. Revista mexicana de biodiversidad 82(1): 237-248.

Moreno, E.A. \& Revuelta, C.M. (2010). La caza de vicuñas en tebenquiche chico (dpto. Antofagasta de la sierra, catamarca). Relaciones de la Sociedad Argentina de Antropología, 35. Disponible en http://sedici.unlp.edu. ar/bitstream/handle/10915/20939/ Documento_completo.pdf? sequence $=1$

Nielsen, C.; Thompson, D.; Kelly, M. \& Lopez-Gonzalez, C. 2015. Puma concolor. The IUCN Red 
List of Threatened Species 2015: e.T18868A97216466. doi:http://dx.doi. org/10.2305/IUCN.UK.2015-4.RLTS. T18868A50663436.en

Nijkamp, P.; Vindigni, G. \& Nunes, P. A. 2008. Economic valuation of biodiversity: A comparative study. Ecological economics 67(2): 217-231. doi:10.1016/j.ecolecon.2008.03.003

Noguera-Urbano, E.A.; Ramírez-Chaves, H.E. \& Torres-Martínez, M.M. 2016. Análisis geográfico y conservación del zorro andino Lycalopex culpaeus (Mammalia, Canidae) en Colombia. Iheringia, Série Zoologia, 106(e2016014), e2016014. Disponible en https://www.researchgate.net/ profile/Hector_Ramirez-Chaves $2 /$ publication/305747841_Analisis geografico_y_conservacion_del_ zorro_andino_Lycalopex_culpaeus Mammalia_Canidae_en_Colombia//57a 0cdf608aeef35741b79cf.pdf

Noss, A.; Villalba, L. \& Arispe, R. 2010. Felidae. Disponible en https://www.researchgate.net/ publication/269709354_Felidae

Pacheco, V. 2002. Mamíferos del Perú. Diversidad y conservación de los mamíferos neotropicales. ConabioUNAM, 503-550. Disponible en http://s3.amazonaws.com/academia. edu.documents $/ 6523411 /$ pacheco_ mammals_peru_2002.pdf?AWSAc cessKeyI $\bar{d}=$ AKIAIWOWYYGZ2Y 53UL3A\&Expires $=1493864096 \&$ Signature $=$ E 8 P XCVWRAGMburi u 1 bUXOS0DS3M\%3D\&responsecontent-disposition=inline $\% 3 \mathrm{~B} \% 20 \mathrm{file}$ name\%3DMamiferos_del_Peru.pdf

Palacios, R. 2007. Manual para identificación de carnívoros andinos. Córdoba, Argentina: Alianza Gato Andino. Disponible en https:// s3.amazonaws.com/academia.edu. documents/32038140/MANUAL_
FINAL.pdf?AWSAccessKeyId=AKIA IWOWYYGZ2Y53UL3A\&Expires $=15$ 02069179\&Signature $=\%$ 2BI1 $\mathrm{rdPnb} \% 2$ FbpC2NHkVcimvyn860g\%3D\&respon se-content-disposition $=$ inline $\% 3 \mathrm{~B} \% 20$ filename\%3 DManual_para_la identificacion

Pinedo, K.; Chávez, A.; Rivera, H.; Pinedo, R. \& Suárez, F. 2014. Frecuencia de Toxoplasma gondii Y Neospora caninum en vicuñas (Vicugna vicugna) de la sierra central peruana mediante las técnicas de inmunofluorescencia indirecta y ELISA indirecta. Revista de Investigaciones Veterinarias del Perú 25(1): 70-76.

Polasky, S.; Costello, C. \& Solow, A. 2005. The economics of biodiversity. Handbook of environmental economics. doi:10.1016/ S1574-0099(05)03029-9

Póveda, R.A. 2006. Recursos naturales. En Oportunidad de un país diferente: próspero, equitativo y gobernable. (págs. 367-391). Banco Mundial.

Prugh, L.; Stoner, C.; Epps, C.; Bean, W.; Ripple, W.; Laliberte, A. \& Brashares, J. 2009. El ascenso del mesopredador. Bioscience 59(9): 779 - 791. doi:10.1525/ bio.2009.59.9.9

Pulgar, J. 1981. Geografía del Perú: Las ocho regiones naturales del Perú. Lima: Editorial Universo.

Rocha, N. \& Rumiz, D. 2010. Didelphidae. Disponible en https://www.researchgate. net/profile/Damian_Rumiz/ publication/265380013_Didelphidae//540 b18d50cf2df04e74948c3/Didelphidae.pdf

Rosado, B.B. 2017. Odocoileus virginianus (Venado Cola Blanca). Historia natural, problemática y alternativas de aprovechamiento. Mentor Forestal 1(1): Disponible en http://revistas.unamad.edu. pe/index.php/mentor/article/view/102

Rowe, R.J. 2009. Environmental and geometric drivers of small mammal diversity along elevational gradients 
in Utah. Ecography 32(3): 411-422. doi:10.1111/j.1600-0587.2008.05538.x

Ruiz-Agudelo, C.A.\& Bello, L.C. 2014. ¿El valor de algunos servicios ecosistémicos de los Andes colombianos?: transferencia de beneficios por meta-análisis. Universitas Scientiarum 19(3).

Rumiz, D.I.; Eulert, C.F. \& Arispe, R. 1998. Evaluación de la diversidad de mamíferos medianos y grandes en el Parque Nacional Carrasco (Cochabamba, Bolivia). Revista Boliviana de ecología y conservación ambiental 4: 77-90.

Rumiz, D.I.; Rivero, K.; Gómez, H.; Sainz, L.; Nuñez, A. \& Wallace, R.B. 2010. Cervidae. Disponible en http://www. icneotropical.org/archivos\%20DEER/ articles\%20members/Damian\%20 Rumiz.pdf

SERNANP. 2011. Plan Maestro del Parque Nacional Huascaran 2010-2015. Lima. Disponible en http://old.sernanp. gob.pe/sernanp/archivos/biblioteca/ publicaciones/PN_HUACARAN/ P D F \% 20 B A J A $\% 20$ P A G $\% 20$ INERIORES.pdf

SINIA. 2014. Listado de especies CITES peruanas de fauna silvestre. Ministerio del Ambiente. Lima: Sistema Nacional de Información Ambiental. Disponible en http://www.minam.gob. pe/diversidadbiologica/wp-content/ uploads/sites/21/2014/02/Especiesde-Fauna-Silvestre-Peruana-en-losAp\%C3\%A9ndices-de-la-CITES1.pdf

Tantaleán, M.; Sánchez, L. \& Salízar, P. 2009. Viscachataenia quadrata Denegri, Dopchiz, Elissondo \& Beveridge, 2003 (Cestoda: Anoplocephalidae) en el Perú. Revista Peruana de Biología 16(1): 129130.

Tlapaya, L. \& Gallina, S. 2010. Cacería de mamíferos medianos en cafetales del centro de Veracruz, México. Acta zoológica mexicana 62(2): 259-277.
Veintimilla, N. 2015. Presencia de enfermedades parasitarias e infecciosas (Leptospirosis, distemper y brucelosis) en zorros andinos (Lycalopex culpaeus) que habitan en los páramos de la Hacienda Antisanilla (Pintag-Ecuador) (Bachelor's thesis, Quito: USFQ, 2015). Obtenido de http://repositorio.usfq.edu.ec/ handle/23000/4211

Villalba, L.; Lucherini, M.; Walker, S.; Cossio, D.; Iriarte, A.; Sanderson, J.; SilleroZubiri, C. 2004. El gato andino: Plan de accion para su conservacion. Alianza Gato Andino. La Paz, Bolivia. Disponible en http://www.gatoandino.org/images/ paginas/pdf/El\%20Gato\%20Andino\%20 Plan\%20Accion\%20Conservacion.pdf

Villalba, L.; Lucherini, M.; Walker, S.; Lagos, N.; Cossios, D.; Bennett, M. \& Huaranca, J. 2016. Leopardus jacobita. The IUCN Red List of Threatened Species 2016: e.T15452A50657407. doi:http://dx.doi. org/10.2305/IUCN.UK.2016-1.RLTS. T15452A50657407.en

Werenkraut, V. 2010. Patrones altitudinales en la diversidad de coleópteros $\mathrm{y}$ hormigas epígeos del noroeste de la Patagonia argentina. Doctoral dissertation, Universidad de Buenos Aires, Facultad de Ciencias Exactas y Naturales. Disponible en http://digital.bl.fcen.uba.ar/Download/ Tesis/Tesis_4785_Werenkraut.pdf

Zorrilla, J. 1993. Tipos de investigación. File investipos 4(4): 45-52. 
Anexo 1. Meta-análisis según especie

\begin{tabular}{|c|c|c|c|c|}
\hline $\begin{array}{l}\text { Nombre } \\
\text { común }\end{array}$ & Nombre científico & Autor & $\begin{array}{c}\text { Año } \\
\text { Publicación }\end{array}$ & Altitud \\
\hline \multirow{5}{*}{ Gato montés } & \multirow{5}{*}{ Oncifelis colocolo } & Lucherini et al. & $(2016)$ & 5000 \\
\hline & & SINIA & (2014) & 2000 \\
\hline & & Díaz et al. & (2002) & 1200 \\
\hline & & Noss et al. & (2010) & $100-4580$ \\
\hline & & Clavijo \& Ramírez & (2009) & $3000-4500$ \\
\hline \multirow{4}{*}{ Muca } & \multirow{4}{*}{ Didelphis marsupialis } & Astua de Moraes et al. & (2016) & 2000 \\
\hline & & Briones et al. & (2015) & 2500 \\
\hline & & Rocha \& Rumiz & (2010) & 3200 \\
\hline & & Gálvez & (1991) & $2000-4000$ \\
\hline \multirow{6}{*}{$\begin{array}{l}\text { Oso de } \\
\text { anteojos }\end{array}$} & \multirow{6}{*}{ Tremarctos ornatus } & Goldstein et al. & (2008) & $250-4750$ \\
\hline & & SINIA & (2014) & $800-3500$ \\
\hline & & Figueroa & (2013) & $1900-3200$ \\
\hline & & Figueroa & (2012) & $1900-2350$ \\
\hline & & $\begin{array}{l}\text { del Moral Sachetti et al. } \\
\text { Figueroa }\end{array}$ & $\begin{array}{l}(2009) \\
(2015)\end{array}$ & $\begin{array}{l}250-4250 \\
3400-4000\end{array}$ \\
\hline & & Figueroa & (2016) & $3000-3650$ \\
\hline \multirow{5}{*}{ Taruca } & \multirow{5}{*}{$\begin{array}{l}\text { Hippocamelus } \\
\text { antisensis }\end{array}$} & Barrio \& Ferreyra & (2008) & $2000-5000$ \\
\hline & & SINIA & (2014) & $3800-5000$ \\
\hline & & Barrio & (2006) & $3500-5000$ \\
\hline & & Rumiz et al. & (2010) & 4900 \\
\hline & & Barrio & (2006) & $2000-3500$ \\
\hline \multirow{6}{*}{ Vizcacha } & \multirow{6}{*}{ Lagidium peruanum } & Bernal & (2016) & 5100 \\
\hline & & Tantaleán et al. & (2009) & 3000 \\
\hline & & Pacheco & (2002) & 4500 \\
\hline & & Lagos & (2012) & 4338 \\
\hline & & Jiménez et al. & (2006) & $2600-4800$ \\
\hline & & Fajardo et al. & (2014) & 4100 \\
\hline \multirow{6}{*}{ Gato andino } & \multirow{6}{*}{ Leopardus jacobita } & Villalba et al. & (2016) & $650-5000$ \\
\hline & & SINIA & (2014) & $3300-4800$ \\
\hline & & Clavijo \& Ramírez & (2009) & $3500-4500$ \\
\hline & & Villalba et al. & (2004) & $3900-4500$ \\
\hline & & Noss et al. & (2010) & $3000-5010$ \\
\hline & & Fajardo et al. & (2014) & 4000 \\
\hline \multirow{7}{*}{ Vicuña } & \multirow{7}{*}{ Vicugna vicugna } & Lichtenstein et al. & (2008) & $3200-5000$ \\
\hline & & SINIA & (2014) & $3800-5000$ \\
\hline & & Dourojeanni & (1968) & 4500 \\
\hline & & Moreno \& Revuelta & (2010) & $3600-4800$ \\
\hline & & Encina \& Iglesias & (2009) & 3000 \\
\hline & & Cajal & (1989) & 3600 \\
\hline & & Aguilar & (2011) & $3000-4600$ \\
\hline
\end{tabular}




\begin{tabular}{|c|c|c|c|c|}
\hline $\begin{array}{l}\text { Nombre } \\
\text { común }\end{array}$ & Nombre científico & Autor & $\begin{array}{c}\text { Año } \\
\text { Publicación }\end{array}$ & Altitud \\
\hline \multirow{6}{*}{ Zorrino, Añas } & \multirow{7}{*}{ Conepatus chinga } & Emmons et al. & $(2016)$ & 4100 \\
\hline & & Gómez-Puerta et al. & (2009) & 4000 \\
\hline & & Jayat et al. & (1999) & 3500 \\
\hline & & Medina et al. & (2012) & $2550-3600$ \\
\hline & & Gallina \& Lopez Arevalo & (2016) & 4500 \\
\hline & & Garzón-Jarrin et al. & (2016) & $3400-4700$ \\
\hline \multirow{5}{*}{$\begin{array}{c}\text { Venado cola } \\
\text { blanca }\end{array}$} & & Medina et al. & (2012) & $2800-3600$ \\
\hline & \multirow{4}{*}{ Odocoileus virginianus } & Rosado & (2017) & $1000-3800$ \\
\hline & & Rumiz et al. & (2010) & $3200-4100$ \\
\hline & & Lavariega et al. & (2012) & $1800-2915$ \\
\hline & & Albuja Viteri & (2007) & 3300 \\
\hline \multirow{4}{*}{ Zorro andino } & \multirow{4}{*}{ Pseudalopex culpaeus } & Lucherini & (2016) & 4800 \\
\hline & & Jiménez et al. & (1996) & 4500 \\
\hline & & Veintimilla & (2015) & $1800-4000$ \\
\hline & & Noguera-Urbano et al. & (2016) & $2000-3700$ \\
\hline \multirow{7}{*}{ Guanaco } & \multirow{7}{*}{ Lama guanicoe } & Baldi et al. & (2016) & 5000 \\
\hline & & SINIA & (2014) & 4500 \\
\hline & & Pinedo et al. & (2014) & $3000-4600$ \\
\hline & & Castillo et al. & (2008) & $1900-5200$ \\
\hline & & Cafrune et al. & (1996) & 3500 \\
\hline & & Huamán Fuertes & (2008) & $3000-4000$ \\
\hline & & Barberena et al. & (2010) & $1000-3600$ \\
\hline \multirow{10}{*}{ Puma } & \multirow{10}{*}{ Puma concolor } & Nielsen et al. & (2015) & 5800 \\
\hline & & SINIA & $(2014)$ & 5000 \\
\hline & & Chimento et al. & (2014) & $3000-5800$ \\
\hline & & Medina et al. & (2012) & $1300-3600$ \\
\hline & & Arias-Alzate et al. & (2016) & $1800-3000$ \\
\hline & & Noss et al. & (2010) & 5000 \\
\hline & & Clavijo \& Ramírez & (2009) & 4100 \\
\hline & & Lavariega et al. & (2012) & $2385-2525$ \\
\hline & & Iriarte et al. & (2013) & $3000-5000$ \\
\hline & & Palacios & $(2007)$ & 3500 \\
\hline
\end{tabular}



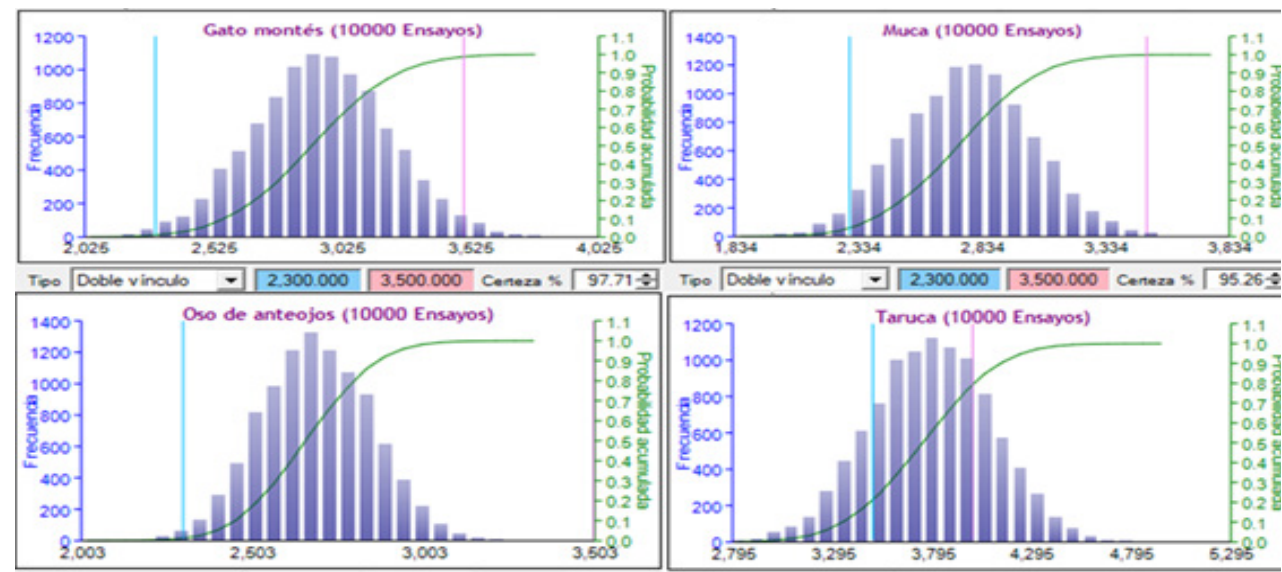

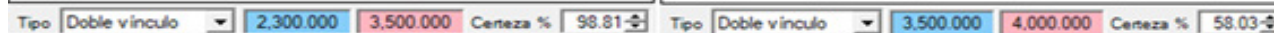
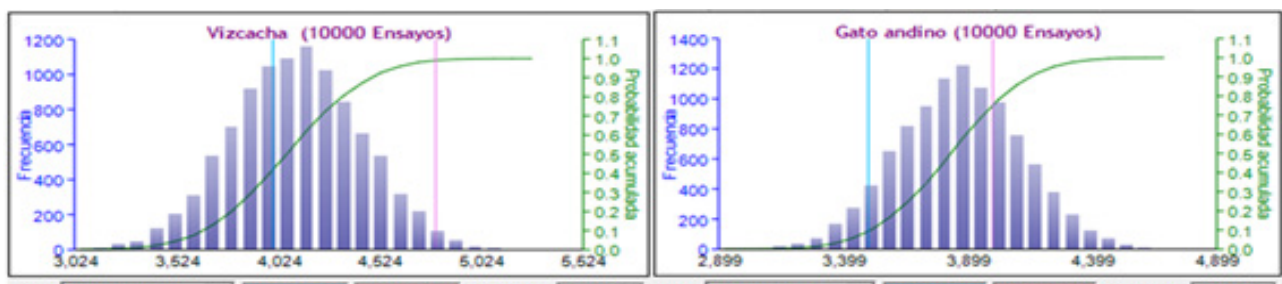

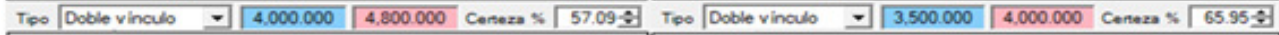

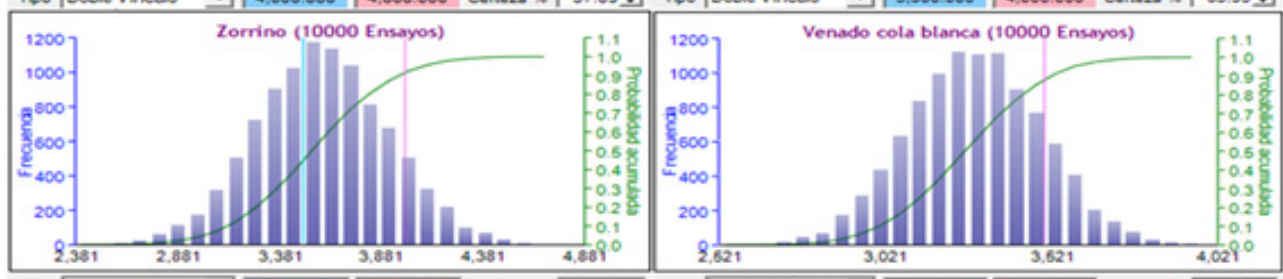

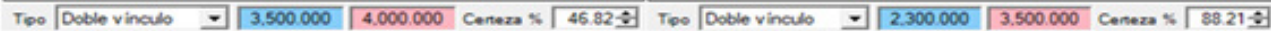

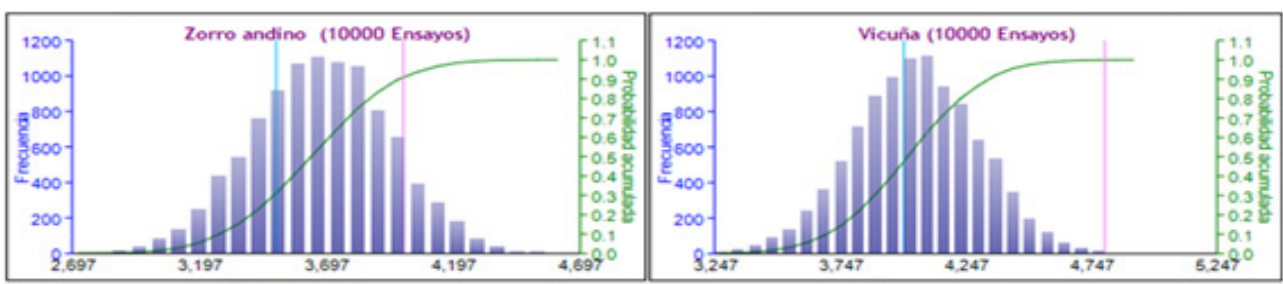

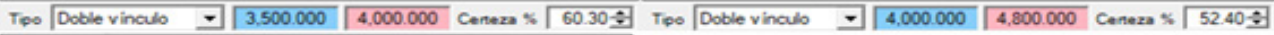

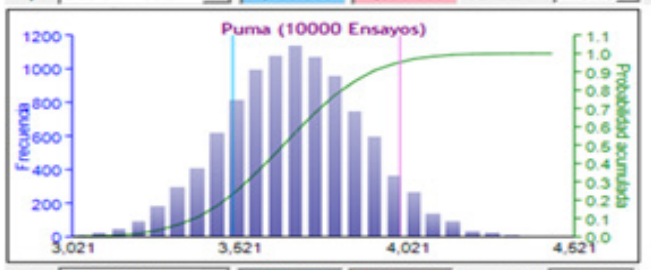

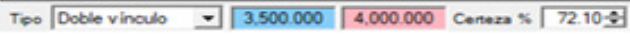

